\title{
Object-Oriented Remote Sensing Image Classification and Road Damage Adaptive Extraction
}

\author{
Xiaoli Liu, Xue Li, Jinggang Li, Qiuliang Wang \\ Key Laboratory of Earthquake Geodesy, Institute of Seismology, CEA, Wuhan, China \\ E-mail: liuxl.j@gmail.com
}

\begin{abstract}
Quick road extraction from earthquake disaster area is very important for the earthquake emergency and rescue. For taking advantage of the multiple data sources and complexity of the contextual environments, an automatic model on the road detection is created by using a number of feature descriptors from damaged roads.The method takes advantage of effective shape, texture, spatial indices to eliminate non-road objects, and then geometry and attribute information, such as length, width, area, damage ratio, damage location of damaged roads, are calculated by registering pre-disaster road vectors and the classification results. Finaaly, the techniques are employed to extract the damaged road section for Wenchuan, China disaster area and Lushan, China disaster area are implemented as examples. The result indicates that this method can improve quickness and efficiency of road damage detection.
\end{abstract}

Index Terms-Remote sensing imagery, object-oriented, adaptive scale, road damage detection

\section{INTRODUCTION}

In recent years, frequent earthquakes, such as the Wenchuan earthquake, the Yushu earthquake, the Lushan earthquake, bring great harm to life and property. After a severe disaster, roads often become impassable because of the serious damage, so emergency transportation for rescue cries for road damage information in disaster area. With the rapid development of the high-resolution remote sensing image, remote sensing technology has substantially enhanced its capacity of the rapid access to road disaster information[1]. Some countries, such as Japan and the United States, have carried out the study and monitoring of the disaster information after the earthquake based on the multi-source remote sensing data that can be rarely affected by the destroy from the earthquake [2-4]. However, research for road damage detection from re-mote sensing images is relatively small. F.Samadzadegan and N.Zarrinpanjeh use pre-event digital vector map and post-event satellite image to extract city road network, and then detect and remove violating objects by fuzzy inference system. Finally, the damage and no-damage objects are marked[5]. W. Yanping presents an object-oriented method of road and damaged road detection which uses many image features synthetically and combines with pre-disaster's GIS vector information[6]. Z. Shaoguang extract most of the roads with weak and broken boundaries by high-resolution RS images[7].
The above methods takes into account the shape parameter, but seldom consider the road texture characteristics before and after the earthquake. In this paper, an object-oriented adaptive extraction method of road disasters is present to get quickly quantitative data of road damages. The method avoids the difficulties and inaccuracy caused by single-phase images after the earthquake, and provide some reference and basis for the post-disaster road damage assessment.

\section{2. IMAGE FEATURES OF POST-DISASTER ROAD}

In the earthquake disaster, there are three main types of roads damage: (1) road deformation, cracking or collapse caused by the earthquake force; (2)road shock or buried by mudslides, landslides and other disasters; (3) road flooded by dammed lakes.

In the remote sensing images, roads usually present the regional feature with a certain length and width. But after the earthquake, the image characteristics of the road have changed in terms of texture and gray. For example, road buried by broken stones and intact road are similar in gray. The traditional method based on gray value, such as road recognition method based on least squares spline curve, edge tracking algorithm, hardly consider texture differences between roads and its background, and shape characteristics of road, therefore classification performance and practicality are often less than ideal. Based on the above conclusions, the characteristics of the gray value, texture, and shape are taken into account.

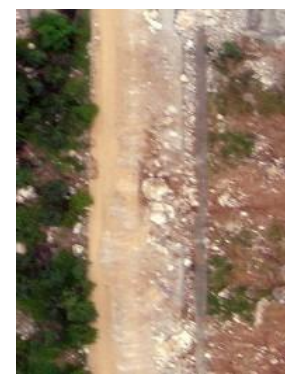

(a)

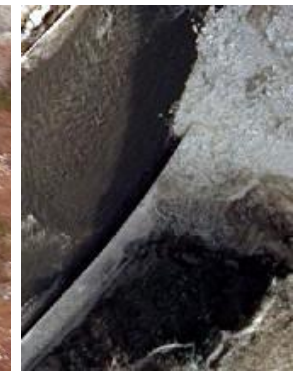

(b)

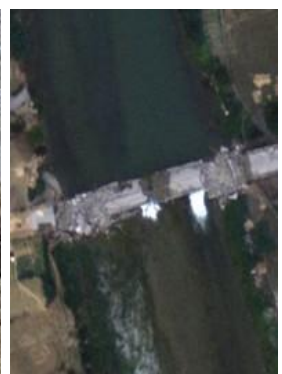

(c)
Fig. 1 The images of the damage road buried by (a) roken stones, (b) landslides, (c)the collapsed bridge 


\section{3. THE OBJECT-ORIENTED ADAPTIVE EXTRACTION OF ROAD DAMAGE INFORMATION}

With the improvement of spatial resolution, image detail is becoming more and more rich, and then the road unable to distinguish by the low-resolution images can discerned now, but more and more non-target noise come along at the same time. Especially in certain conditions of the secondary disasters (such as dammed lakes and landslides), the road and other surface features with similar gray values are often difficult to distinguish. This paper presents an object-oriented road damage detection method. Firstly, based on pixel, multi-scale segmentation technology is adopted to get image objects, this is road objects. Secondly, road information are extracted by the object-oriented and multi-scale classification method with some typical parameters, such as spectrum, texture, structure, distance, topology and direction of road objects, as shown in Fig. 2.

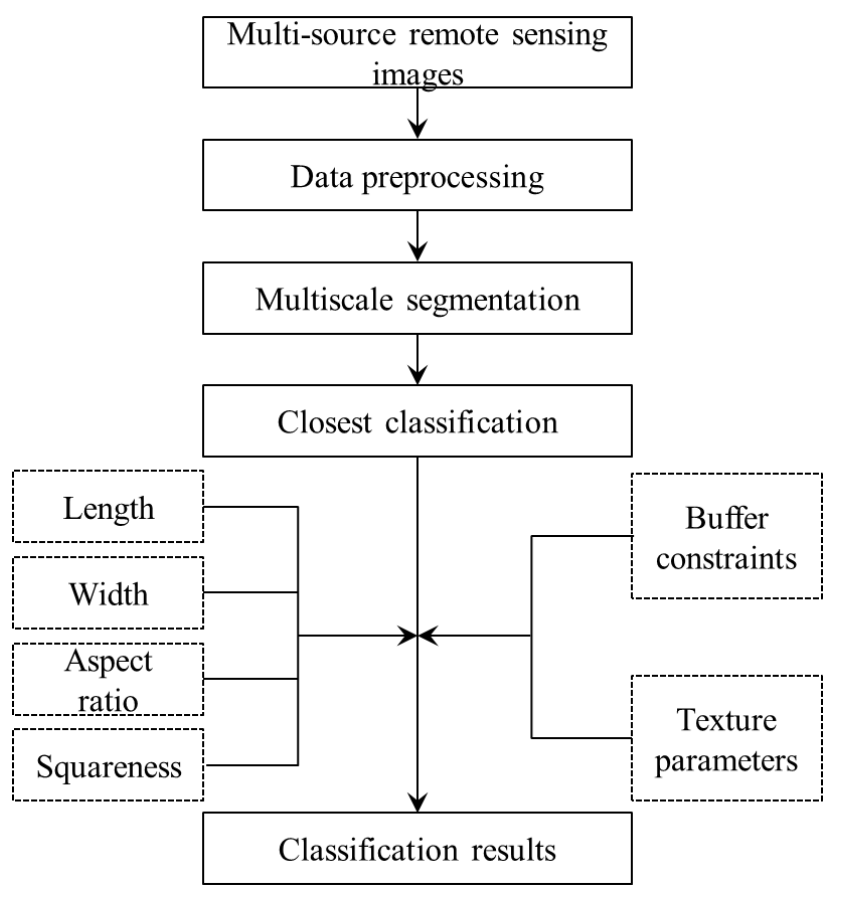

Fig. 2 Flowchart of road damage detection

\section{A. Segmentation scale}

Segmentation scale is a minimum threshold limiting to the splited object heterogeneity, and vary along the size of image resolution[8]. Generally speaking, the higher the resolution, the more rich details of the image features, and the greater pixel heterogeneity, and then more sensitive to the scale changes. In this case, initial segmentation scale should be set smaller, and the scale should be gradually increased if the segmentation results is not ideal. On the contrary, the lower the resolution, the more blurred the image, less sensitive to the scale changes, and then initial segmentation scale should be set larger. In multiscale segmentation process of road images, if the scale is too large, the road and background may be divided into the same object, resulting in misclassification; if the scale is too small, roads having the shape characteristics may be divided into several fragmented spots, and then affect the integrity of the road. Therefore small noise (such as vehicles, white centerline) implicit in the road objects is extracted and undermines the integrity of the road objects.

\section{B. Object heterogeneity}

Object heterogeneity includes spectral heterogeneity and shape heterogeneity, and the latter can be divided into smoothness degree used to ensure the objects with smooth boundary, and compact degree heterogeneity used to distinguish the compact target and non-compact target with the smaller differences. Image object heterogeneity is calculated by (1):

$$
f=w_{a} \cdot h_{\text {spectral }}+w_{b} \cdot h_{\text {smooth }}+w_{c} \cdot h_{\text {compact }}
$$

Where $w_{a}, w_{b}$ and $w_{c}$ respectively stand for user-defined spectral weight, smoothness degree weight and compact degree weight, while $h_{\text {spectral }}, h_{\text {smooth }}$ and $h_{\text {compact }}$ are respectively spectrum heterogeneity, smoothness degree heterogeneity and compact degree heterogeneity. The three heterogeneities are expressed as (2), (3) and (4):

$$
\begin{aligned}
& h_{\text {spectral }}=\sum_{s} \omega_{s} \cdot \sigma_{s} \\
& h_{\text {smooth }}=\frac{l_{1}}{\sqrt{n}} \\
& h_{\text {compact }}=\frac{l_{1}}{\sqrt{b}}
\end{aligned}
$$

Where $\sigma_{s}$ is the standard deviation, $\omega_{s}$ is the spectrum weight of each level, $l$ is the perimeter of the image object, $n$ is the size of the image object, and $b$ is the perimeter of the enclosing rectangle of the image object.

\section{Structure parameters}

The spectral characteristics of the road on high-resolution remote sensing images is relatively uniform of the internal and contrast completely to adjacent areas[9]. In addition, the road also has the following features. (1) geometric features: the road generally have a large aspect ratio and a limited curvature. (2)topological features: the road doesn' t suddenly interrupted, intersect with each other, and form a road network. With the above features, classification results be amended by the objectoriented technology. For example, the image objects are constraints with rectangular shape parameter, such as the length, the width and the aspect ratio in the classification process. Roads are effectively separate with buildings, wells and other surface features, and then the results are usually ideal. 


\section{Texture features}

When roads are buried by crushed stones caused by landslides, the gray features of crushed stones are very similar to the intact roads, wherefore they are divided into the same image object. In the following closest classification process, damaged roads will not be able to distinguish only with the shape parameters and road buffer constraints. It's worth noting that, the textural features of crushed stones is very different to the intact road. Therefore, this classification process must fully utilize differences among the spectral features, geometric features and textural features[10], and finally to analyze and classify the damaged and undamaged objects by turn. Clearly this method is better than the traditional classification method which is based on the pixels. Experiments show that the classification results are ideal with texture parameter constraints, such as haralick operator.

\section{E. Weight values}

Spectral information is the most abundant on the images. Therefore spectral features are the main factor, and shape features are auxiliary factors in the classification process. Typically, the weight of spectral features is in the range of 0.60.8 , the weight of shape features is accordingly in the range of $0.2-0.4$. If the image resolution is low, the shape information is not rich enough, it is necessary to set a higher spectral weight. In the paper, the images used in experiments are with higher resolution, the details of image objects are rich, thus a larger weight of shape features is set in the range of 0.3-0.4.
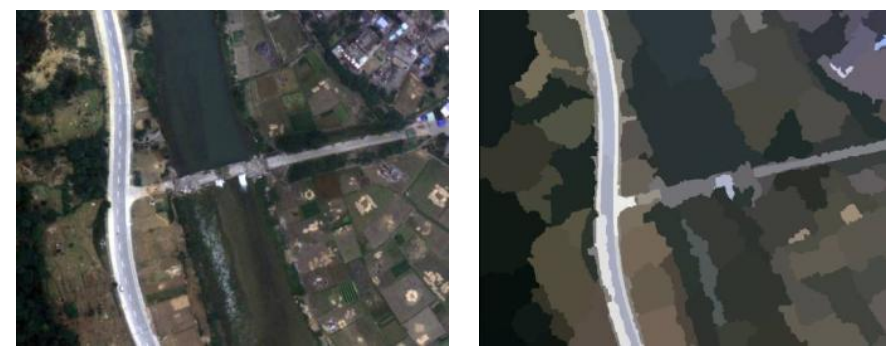

Fig.3 Post-earthquake high-resolution images and multi-scale segmentation results
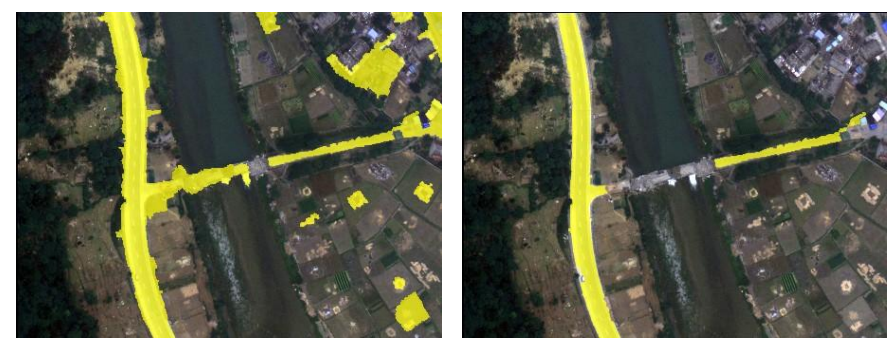

Fig. 4 The results of the initial classification and the constraint classification

\section{F. Road damage information extraction}

By the multi-scale constraint classification, intact roads, damaged roads and background can be distinguished clearly, but the quantized values of length, width, damage ratio, damage location of damaged roads. In the paper, a method is adopted to calculate geometry and attribute information, such as length, width, area, damage ratio (There are three cases: intact, partially damaged, completely destroyed.), damage location (image coordinates or latitude and longitude) of damaged roads, by registering pre-disaster road vectors and the classification results.

\section{EXPERIMENTS AND RESULTS}

At 8:02 (Beijing Time) on April 20, 2013, a catastrophic earthquake with Ms 7.0 struck Lushan County in Sichuan Province, China. This earthquake triggered abundant geohazards such as landslides, collapses and dammed lakes[11] These earthquake induced geohazards causes serious damage to the road infrastructure, which has bad influenceon the disaster-reliefwork after the earthquake. In consideration of this, using the severe calamity area in the Lushan earthquake zone as an example, this paper verifies the efficiency of this method, having respectively selected the SPOT-5 images and the unmanned air images of Lushan, China area on April 21th, 2013 and the SPOT-5 images and the unmanned air images of Wenchuan, China area on May 16th, 2008 as experimental data. In combination with the above mentioned features, the paper fulfilled the information extraction of road features based on road features and the identification of the damaged road section.

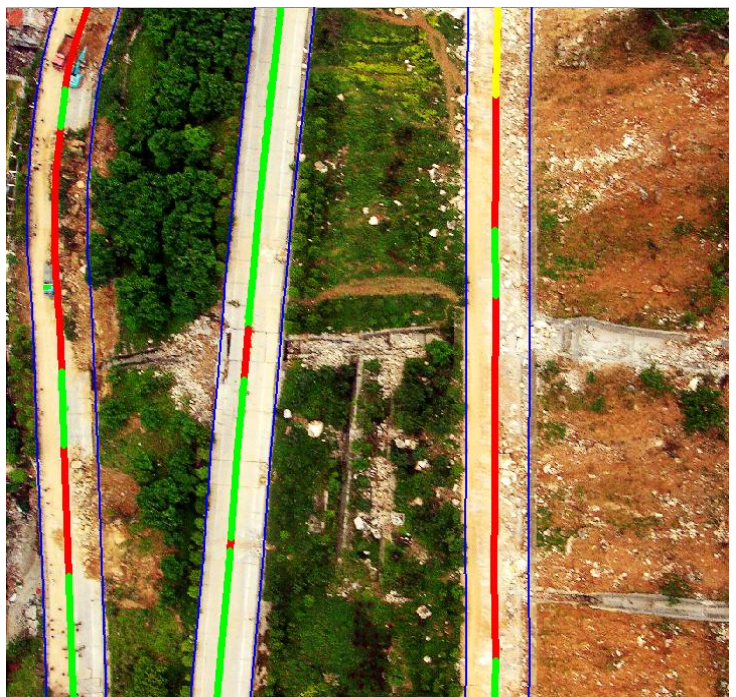

(a)Roads extraction of Wenchuan 


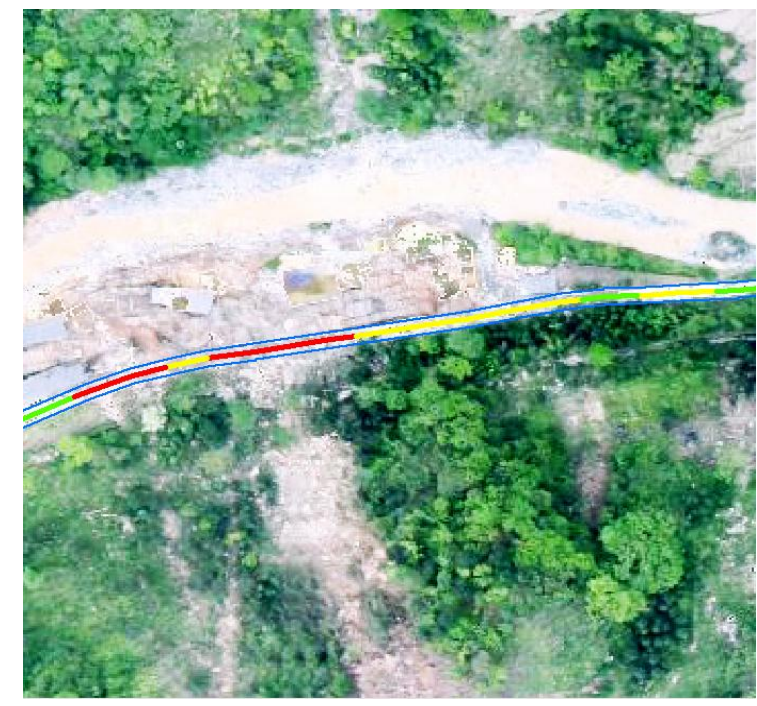

(b) Roads extraction of Lushan

Fig. 5 Damaged road section (Red, yellow, green, blue respectively stand for completely damaged roads, partially damaged roads, intact road and road buffers.)

Fig. 5 is the results of the road information extraction. This is nearly consistent with the visual identification. In the postearthquake images of Wenchuan, China area, there are 7 completely damaged road sections, 1 partially damaged road section, and 8 intact road sections . As shown in Fig. 5(a). In the post-earthquake images of Lushan, China area, there are 2 completely damaged road sections, 3 partially damaged road sections, and 3 intact road sections. As shown in Fig. 5(b).

\section{CONCLUSION}

The paper presents an object-oriented method of road and damaged road detection which uses many image features synthetically and combines with pre-disaster's road vector information. The advantages of this method are that damaged road section can be detected clearly. Furthermore, tasks can be implemented even when there is a lack of pre-disaster image. Must be noted that the green belts of the roads and traveling vehicles on the roads are not divided into road objects in the multi-scale segmentation process. This case may cause a misclassification result of the closest classification. For this reason, textural features must be considered.

\section{ACKNOWLEDGMENT}

This work was supported by the Director Fund (IS201116002) from Institute of Seismology, China Earthquake Administration.

\section{REFERENCES}

[1] C. Shi-rong, M. Hai-jian, F. Yi-d,XU et al. "Road Damage Assessment from High Resolution Satellite Remote Sensing Imagery in Wenchuan Earthquake," Journal of Remote Sensing, 2008, vol. 12, pp. 949-955.

[2] Y. Miyagi, T. Ozawa and M. Shimada. "Crustal deformation associated with an M8.1 earthquake in the Solomon Islands, detected by ALOS/PALSAR," Earth and Planetary Science Letters, 2009, vol. 3-4, pp. 385-391.

[3] O. Nioki and Y. Furmio. "Photo-interpretation of building damage due to earthquakes using aerial photographs," Proc of 12 WCEE, New Zealand Society for Earthquake Engineering, 2000, vol.15, pp.1906.

[4] G. Peltzer, F. Crampé and P. Rosen. "The Mw 7.1, Hector Mine, California earthquake: surface rupture, surface displacement field, and fault slip solution from ERS SAR data," Earth and Planetary Science, 2001, vol. 9, pp. 545-555.

[5] F. Samadzadegan and N. Zarrinpanjeh. "Earthquake destruction assessment of urban roads network using satellite imagery and fuzzy inference systems," Proc. The International Archives of the Photogrammetry, Remote Sensing and Spatial Information Sciences, 2008, pp. 409-414.

[6] W. Yanping, J. Jiyi, L. Lingling. "Road damage detection from high resolution remote sensing," Computer Engineering and Applications, 2012, ol.3, pp.173-175.

[7] Z. Shaoguang, X. Yong. "To Extract Roads with No Clear and Continuous Boundaries in RS Images," Acta Geodaetica et Cartographica Sinica, 2008, vol.3, pp.301-307.

[8] H. Jingang, Z. Xiaodong, S. Xin, Z. Chan. "A Method of Road Extraction in High-Resolution Remote Sensing Imagery Based on Object-oriented Image Analysis," Remote Sensing Technology and Application, 2006, vol. 3, pp.184-188.

[9] M. Ravanbakhsh, C. Heipke, K. Pakzad. "Knowledge-based road Junction Extraction from High-resolution Aerial Images," Urban Remote Sensing Joint Event, 2007, pp.1-8.

[10] J. Senthilnath, M. Rajeshwari, and S. N. Omkar. "Automatic road extraction using high resolution satellite image based on texture progressive analysis and normalized cut method," J. Indian Soc. Remote Sens, 2009, vol. 37, pp. 351-361.

[11] April 20, 2013 M7.0 earthquake in Sichuan Province Lushan special coverage. http://www.cea.gov.cn/publish/izhenj/468/553/100342/100342/ index.html. 